\title{
Bericht über das praktische Studiensemester bei CargoLifter Development GmbH, Werft Briesen-Brand
}

\author{
Alfred Hentschel, Marco Heyn, Stephan Wojcik
}

Es gibt mehrere Gründe, diesen Bericht über das Praxissemester als wissenschaftlichen Beitrag zu verfassen. Einmal stellt das Unternehmen und vor allem das Erzeugnis einen außergewöhnlichen Untersuchungsgegenstand dar, der neben überschaubaren ingenieurwissenschaftlichen Aufgaben auch in der Produktionsvorbereitung einen hohen Anteil Pionierarbeit beinhaltet, allein was die technische Beschaffenheit und die Größenordnung des Erzeugnisses anbelangt. Andererseits sind die Praktikanten des Studienganges Wirtschaftsingenieurwesen mit sehr komplexen Anforderungen konfrontiert worden, die an sich ihrer Ausbildungsrichtung entsprechen, aber daneben waren Sie im Hinblick auf Teamfähigkeit, soziale Aspekte und selbst handwerkliche Tätigkeiten eben auch gefordert. Dass die Praktikumsaufgabe nicht in dem ursprüinglich geplanten Zeitraum abgeschlossen werden konnte, ist dieser Komplexität geschuldet. So erfährt die Aufgabe eine beabsichtigte studienbegleitende Fortsetzung im 6. und 7. Semester und mit Erweiterung bzw. Präzisierung vielleicht im Diplomsemester. Dadurch wird der enge Kontakt zwischen Unternehmen und Hochschule über größere Studienabschnitte gepflegt. Vor allem erhält die Ausbildung im Hauptstudium einen planmäßigen dualen Charakter, was letztlich für den Studierenden außer bei der Vermittlung von Lehrinhalten (einschl. Labortätigkeit) eine geeignete Methode zum Kennenlernen seines Tätigkeitsfeldes darstellt und somit die Vermittelbarkeit und den Berufseinstieg in das Unternehmen für beide Seiten vereinfacht.

\section{Firmenvorstellung}

Die Vision eines Transportluftschiffes ist Anfang der 90er Jahre aufgekommen, und der CargoLifter ist ins Leben gerufen worden. Das Grundprinzip des Transportluftschiffes basiert auf der Technologie „Leichter als Luft“. Durch das ständige Anwachsen des Transportaufkommens, speziell im Bereich des Schwerlasttransportes, wuchs der Wunsch nach einem alternativen Transportmittel.

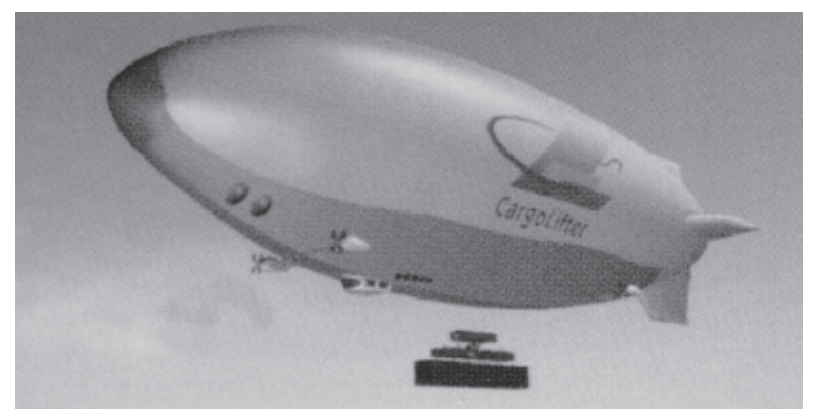

Es soll schwere und sperrige Lasten über große Distanzen transportieren und dabei einen möglichst geringen Landeplatzbedarf haben. Auf dieser Basis entstand die Idee, ein Transportluftschiff zu entwickeln.

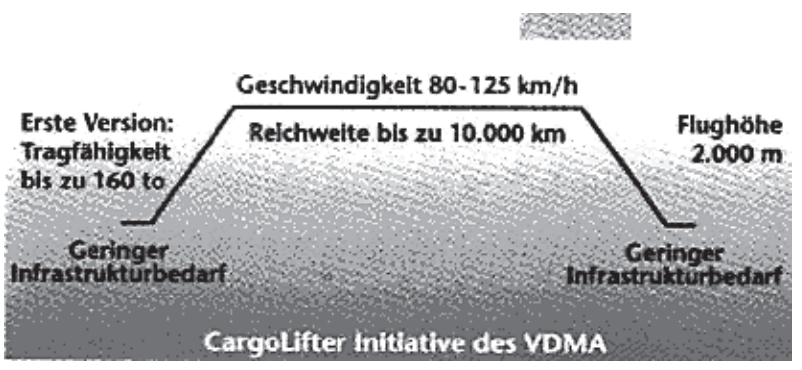

Mit der Gründung der CargoLifter AG im Jahr 1996 ist die industrielle Luftschifffahrt in Deutschland wieder ins Leben gerufen worden. Das Unternehmen wurde am 01.09.1996 gegrüindet und hat seit Herbst 1999 den Sitz in Berlin.

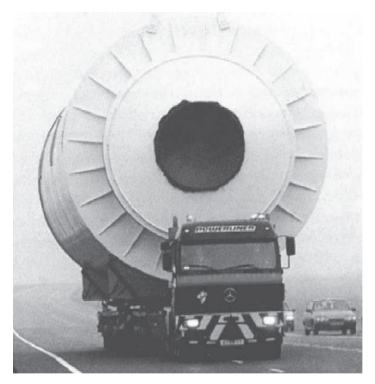

Die Kerngesellschaft des Konzerns bildet die Development $\mathrm{GmbH}$. Sie hat sich die Entwicklung und den Bau des CL 160 auf die Fahne geschrieben. Das Betätigungsfeld der Development $\mathrm{GmbH}$ ist das Werftgelände in Briesen-Brand. Dieser ehemalige Militärflughafen der Roten Armee, $60 \mathrm{~km}$ südlich von Berlin gelegen, umfasst eine Fläche von ca. 326 ha. Auf diesem Areal arbeiten mehr als 240 Menschen, überwiegend Ingenieure (Stand: 29.02.00), aus über 10 Nationen.

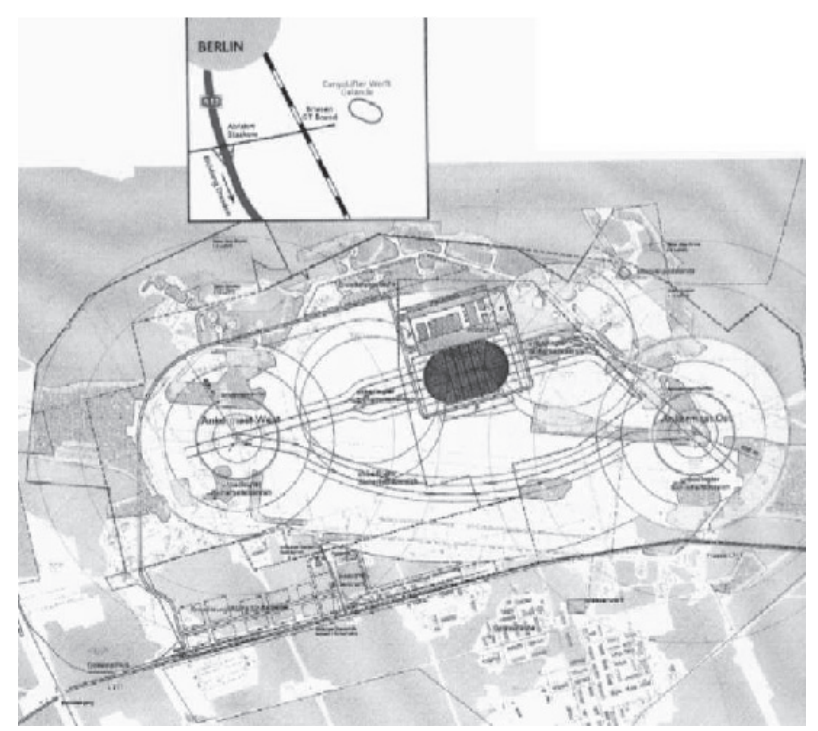


Die Werfthalle ist das Zentrum der Produktionsstätte des CL 160. Am 02. Mai 1998 war der erste Spatenstich, Baubeginn nur kurze Zeit später im August 1998. Sie ist die größte freitragende Halle der Welt. Die Abmessungen sind gewaltig, eine Länge von $360 \mathrm{~m}$, eine Breite von 210 m und ein Höhe von 107 m sind kaum vorstellbar. Der Produktionsstart ist für Herbst 2000 vorgesehen. In diesem Hangar wird das größte Luftschiff der Welt entstehen, mit einer Gesamtlänge von 260 m, 65 m im Durchmesser, einem Hüllenvolumen von $550.000 \mathrm{~m}^{3}$ (gefüllt mit nicht brennbarem Helium) und einer Oberfläche von $50.000 \mathrm{~m}^{2}$. Es wird für Transporte bis $160 \mathrm{t}$ Nutzlast ausgelegt sein. 2002 werden sich die Hangartore öffnen und der erste Prototyp CL 160 P1 wird den Hangar verlassen. Die Serienproduktion nimmt 2004 ihre Arbeit auf.

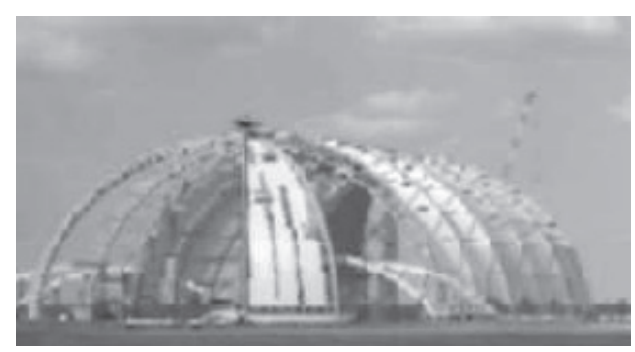

Werfthangar, Stand Juni 2000

Es besteht ein Marktpotential von 200 Transportluftschiffen auf der Welt, so dass sich eine Jahresproduktion von 4 Luftschiffen ergeben wird. Der Bedarf an Luftschiffen errechnet sich aus dem herausgefilterten Marktpotenzial von 30 Mio. Fracht-Tonnen. Davon nimmt man $10 \%, 3$ Mio. Tonnen, als Transportvolumen für den CargoLifter an. Dabei ist noch nicht berïcksichtigt, dass sich durch das neue Transportmittel neue Märkte eröffnen werden.

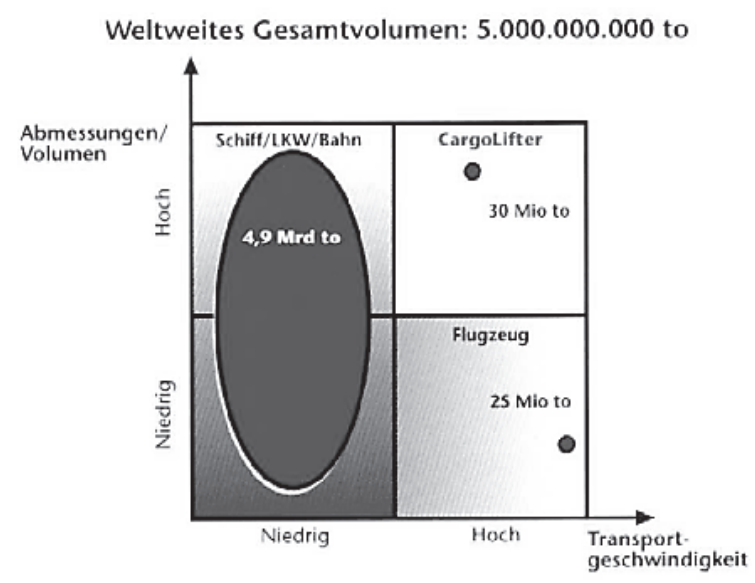

CargoLifter ist im Verhältnis zum Weltmarkt in einem kleinen Marktsegment angesiedelt. Aber diese Positionierung ist ausgesprochen attraktiv. Es ist eine Ergänzung zum Flugzeug und nicht als Konkurrenz zu sehen.

\section{Die Zielmärkte}

Sein Einsatz wird nicht auf Kontinente begrenzt sein, vielmehr wird ein Netz um den ganzen Globus gespannt werden, und so könnte man den Luftraum als „Ozean der Luftschiffe" betrachten.

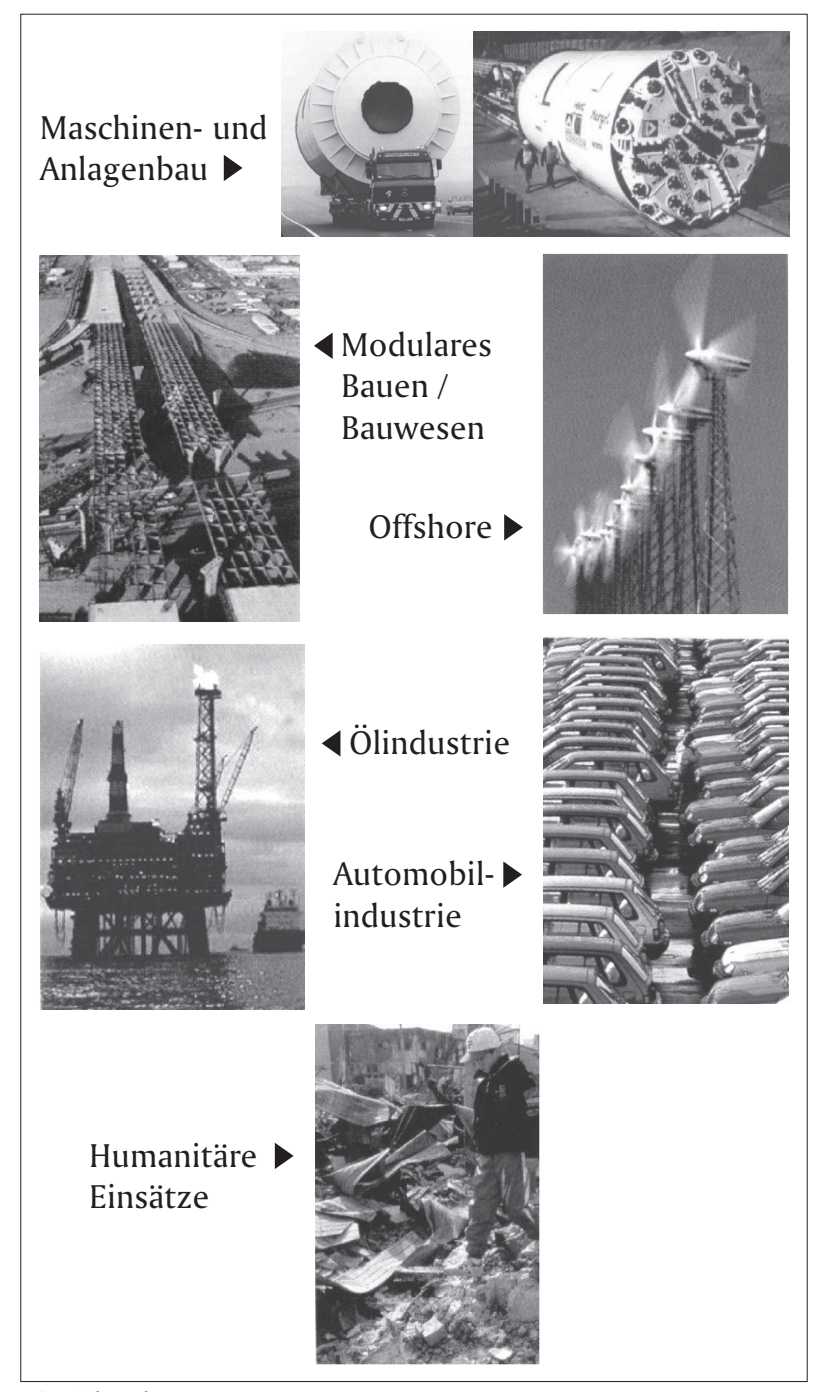

Die Zielmärkte

\section{A new Dimension of Transportation}

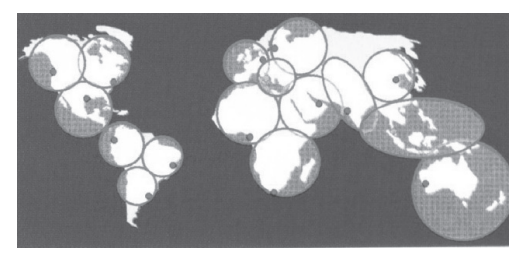

4 Operationsradien

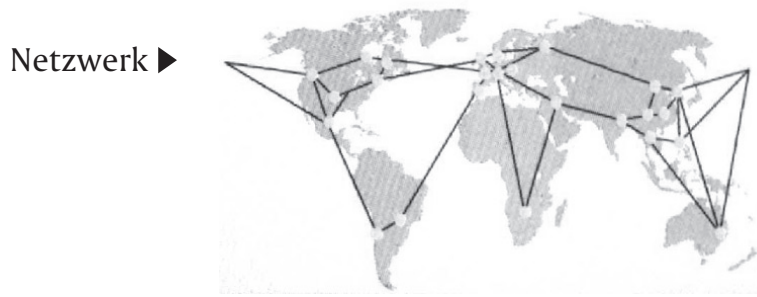

\section{Aufgabenstellung}

Planen und Bauen eines Luftschiffes (Produktionsmodell) im Maßstab 1:16 oder 1:18.

Vorgehensweise: Aufstellen eines Idealzeitplanes über den Zeitraum unserer Tätigkeit im Unternehmen. 


\section{Idealzeitplan}

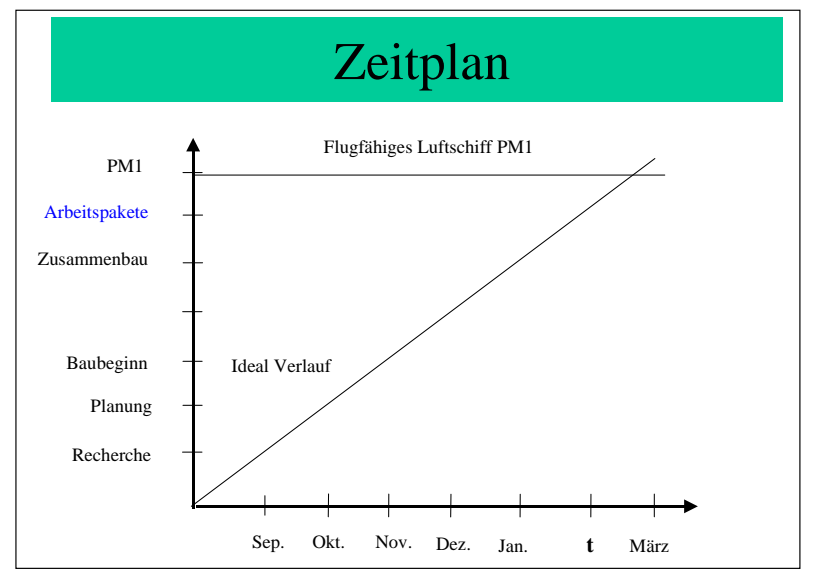

\section{Kurzbeschreibung des Luftschiffes}

\section{Einteilung der Luftschiffe}

Nachdem wir den Zeitplan aufgestellt hatten, gingen wir daran, uns einen groben Überblick über Luftschiffe zu verschaffen. Dabei stellte sich uns die Frage, ob und welche Unterschiede es gibt, ob alle Luftschiffe Zeppeline sind?

Wir sind zu den drei Aussagen gekommen, dass Luftschiffe in drei Typen eingeteilt werden können.

1. Starres Luftschiff

$\Rightarrow$ Zeppelin (Starres Luftschiff/LZ 129 Hindenburg)

2. Blimb Luftschiff

$\Rightarrow$ Luftschiff mit Gondel (Prinzip Ballon)

3. Kiel Luftschiff

$\Rightarrow$ CL 160 (Halbstarres Luftschiff)

\section{Komponenten des Luftschiffes}

Nach der Einteilung der Luftschiffe in diese drei Typen begannen wir mit der Vorbereitung zur Gewinnung von Informationen uiber den CL 160. Wir erkundigten uns, aus welchen Komponenten das Luftschiff bestehen wird. Dies sind:

- Envelope/Hülle,

- Propulsions/Antriebe,

- Keel/Kiel,

- Nose Cone/Nasenkappe,

- Fins/Leitwerke,

- Systems/Systeme.

Den Überblick verschafften wir uns durch die zur Verfügung gestellten Unterlagen und durch Gespräche mit Kollegen. Bei dieser analytischen Vorgehensweise waren wir natuirlich nicht am Ende unserer Recherche. Nun galt es, die grobe Einteilung weiter zu verfeinern. Wir setzten uns in einer Art „Brain-Storming“ zusammen und erarbeiteten die fuir uns wichtigen Informationen, welche notwendig waren, um mit der Planung des Produktionsmodells zu beginnen.

Wir formulierten die Fragen und fügten sie zu einem Formular zusammen. Das Formular mailten wir vorab an die Abteilungsleiter, um den Informationsfluss zu beschleunigen. Nach Terminvereinbarung, Auswertung und Aufbereitung der Daten erfolgte ein Meeting mit Herrn von Kessel (Director of Production), bei dem das erste Resümee unserer Arbeit gezogen werden konnte.

\section{Planung für Hangar-Nachbau}

Resultat des Meetings war, den Shelter 3232 auf die angegebenen Maße aus der Zeichnung zu überprüfen, diese mit den Maßen vom Werfthangar zu vergleichen. Das Ergebnis dieser Untersuchung war, dass wir einen Hangar im Maßstab 1:16 nachbauen konnten.

Es galt, die Nachbauvarianten aufzustellen und unter den folgenden Gesichtspunkten zu prüfen:

- Kosten (Budgetrahmen),

- Arbeitsintensität (Zeit),

- Bedarf an Fachkräften (Machbarkeit),

- Ruickbau (Umwelt).

Am 24.09.99 wurde die Entscheidung für die Variante „PE-Rohr einfach“ besiegelt. Sie war eine von vier weiteren Varianten (Holz, Styropor, PE-Rohr gerippt und Schaumbögen aus einer Form). Des weiteren benötigte der nachgebaute Hangar eine Kranbahn. Auch diese Daten skalierten wir herunter bis auf die zu hebenden Lasten. Der Grund dafuir ist, dass nach Beendigung des Projektes PM1 die Kranbahn weiter fuir andere Zwecke im Unternehmen genutzt werden soll. Wir beschäftigten uns mit der Aufstellung der Eckdaten für die Angebotsabgabe der Firmen und bereiteten die eingehenden Angebote für die Auswertung und die daraus folgende Vergabe vor. Unter Berüicksichtigung unseres Zeitplanes legten wir den Montageendtermin bei der Auftragsvergabe fest.

Die Montageüberwachung und die Kranbahnabnahme fielen ebenfalls in unseren Verantwortungsbereich. Die Montage verzögerte sich um eineinhalb Wochen durch interne Probleme des Auftragnehmers (Maschinenschaden an der Biegemaschine) und durch Annahme von nicht vorhandenen Voraussetzungen (Betonstärke, -qualität / Montagefußboden) des Auftraggebers.

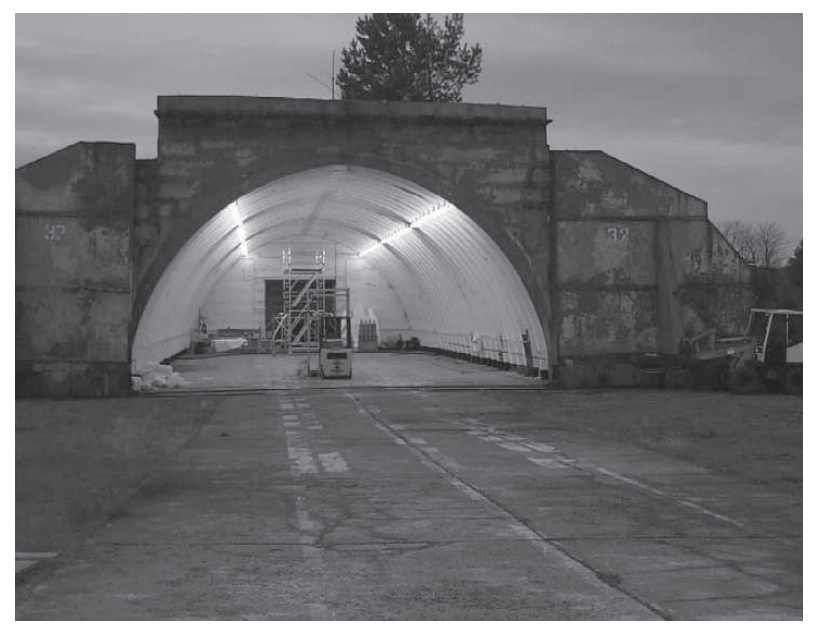

Shelter 3232 


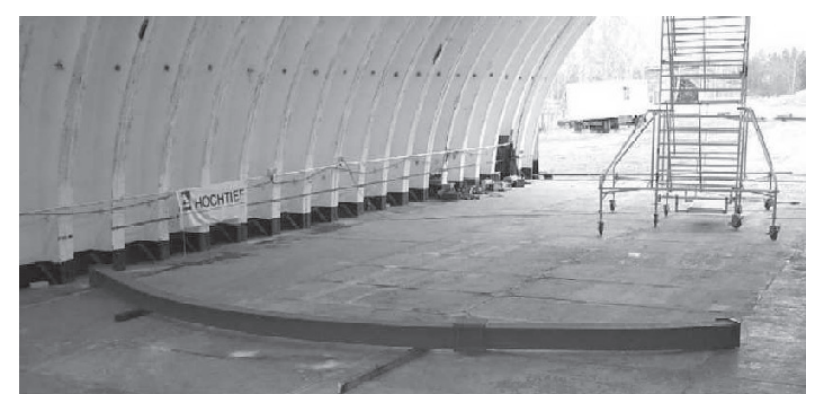

Erste Kranbahnteile werden eingelagert

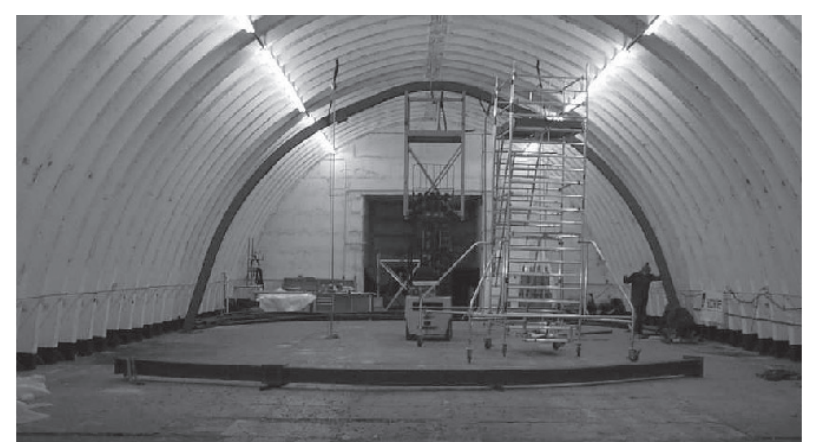

Das erste Portal steht

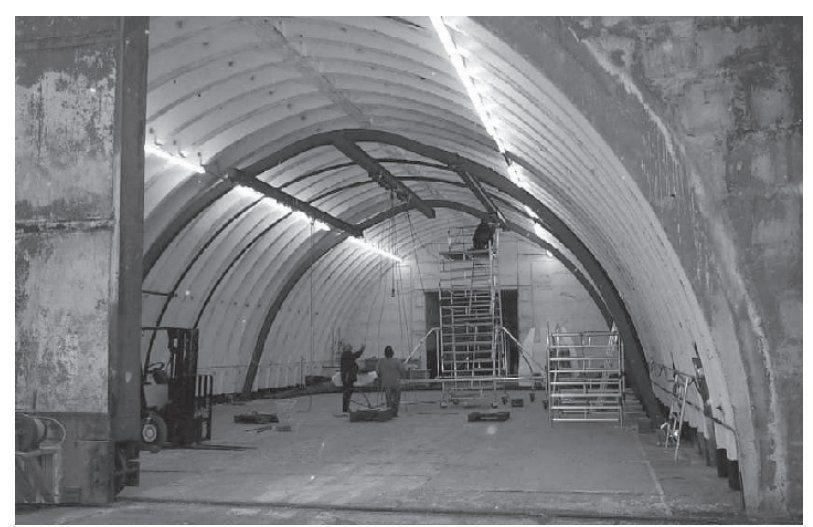

Vorbereitung zur Abnahme vom TÜV

Nach Vergabe des Auftrages der Kranbahn widmeten wir uns der detaillierten Planung zum Nachbau des Hangars. Es mussten der Materialbedarf und Lieferzeiten, evtl. Maschinenbedarf (PE-Schweißmaschine, Hammerbohrmaschine, Propangasbrenner) ermittelt und auf den Montagezeitpunkt abgestimmt werden.

\section{Verzug zeichnet sich ab!}

Bei der ständigen Aktualisierung des Zeitplans (SollZustand) ist uns eine Abweichung und daraus folgend ein zeitlicher Verzug verdeutlicht worden.

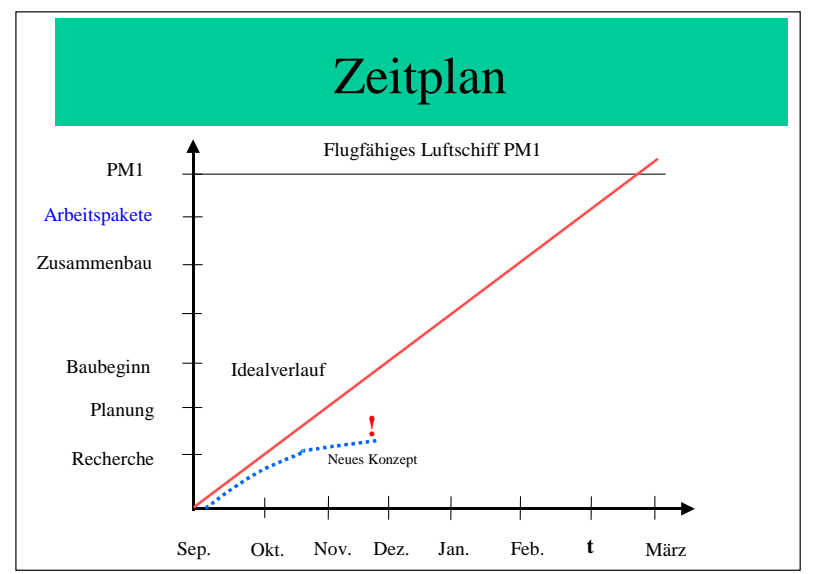

Die Ursachenanalyse ergab, dass der Verzug nicht nur durch die Kranbaufirma hervorgerufen, sondern auch ein großer Teil intern verursacht wurde. Wir mussten das Projekt, so wie wir es am Anfang ausgelegt hatten, überdenken. Es war am Beginn nicht der volle Umfang des Projektes ersichtlich, demzufolge hatten wir zu wenig Ressourcen (Manpower, Planer) eingeplant.

Ursachenbehebung:

- Splitten der Aufgaben / Arbeitspakete

- Schaffen einer internen Aufbau- und Ablauforganisation

- Einbeziehung aller System-Ingenieure

\section{Neues Konzept}

Zur Vermittlung des neuen Konzepts, welches mit dem Praktikumsbetreuer vom Unternehmen abgestimmt wurde, haben wir ein Meeting mit allen Beteiligten zur Vermittlung des Inhalts einberufen.

Die Resultate des neuen Konzepts waren deutlich in unserm Projektplan spürbar. Durch den persönlichen Einsatz und die tatkräftige Unterstuitzung von den Systemingenieuren waren sichtliche Erfolge zu verzeichnen.

Die Planung der Nose Cone und die daraus folgende Materialbestellung sind die ersten Anzeichen für das Greifen des Konzeptes gewesen. Mitte Dezember konnten wir mit dem Bau der Nose Cone beginnen.

\section{Nose Cone / Nasenkappe}

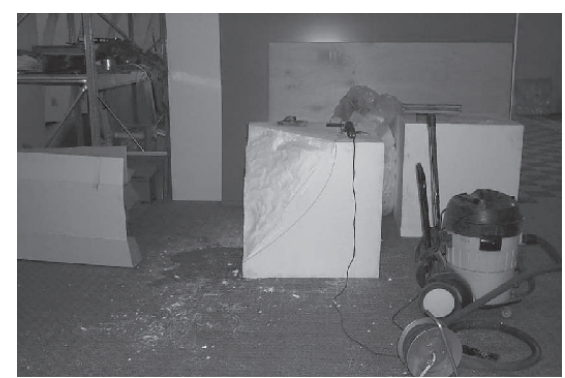

Die Form wurde aus einem $1 \times 1 \times 1 \mathrm{~m}$ großen Styroporblock gefräst
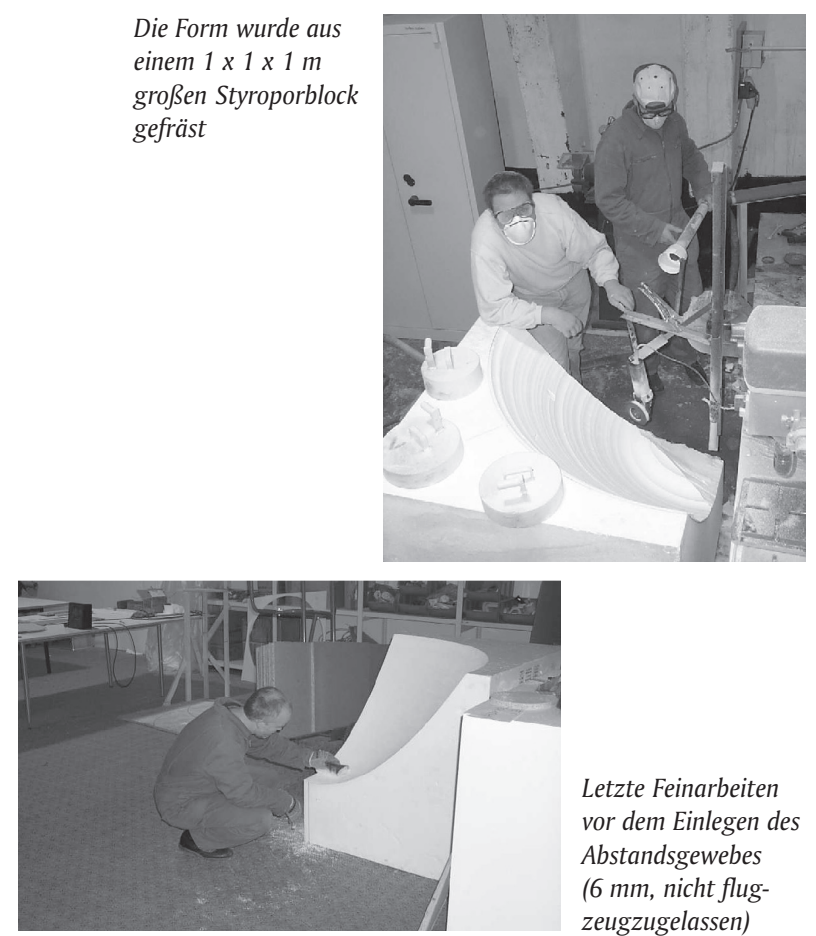

Letzte Feinarbeiten vor dem Einlegen des Abstandsgewebes (6 mm, nicht flugzeugzugelassen) 


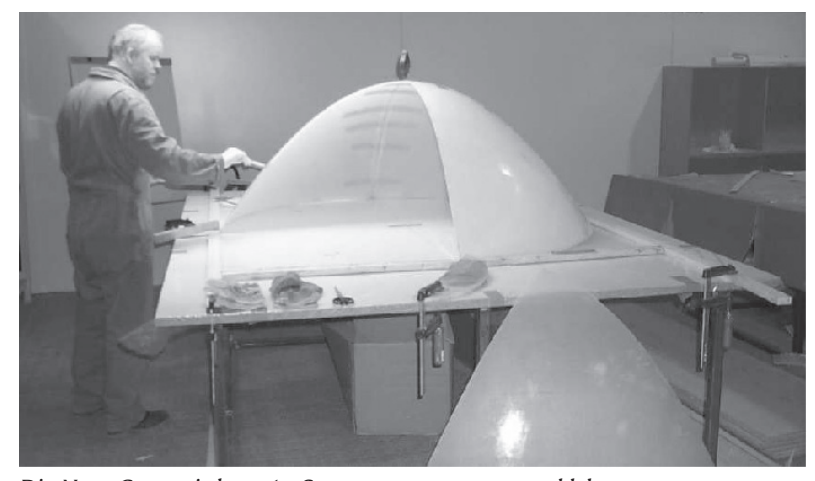

Die Nose Cone wird aus 1/4 Segmenten zusammengeklebt.

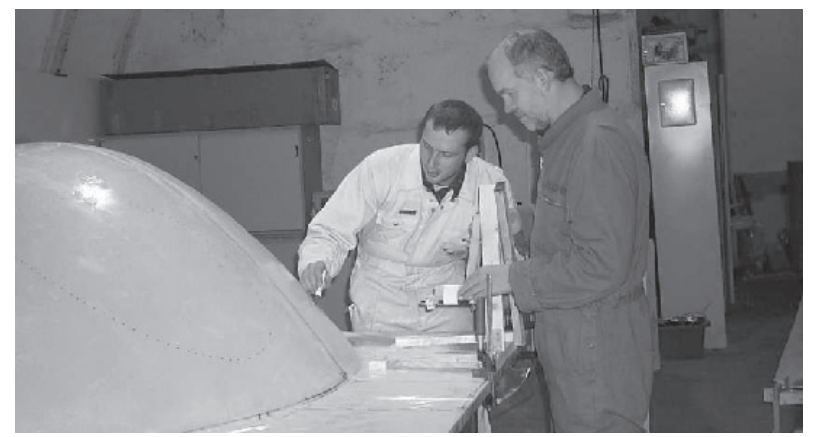

Die zusammengefügten 4 Segmente bilden die Basis der Nose Cone.

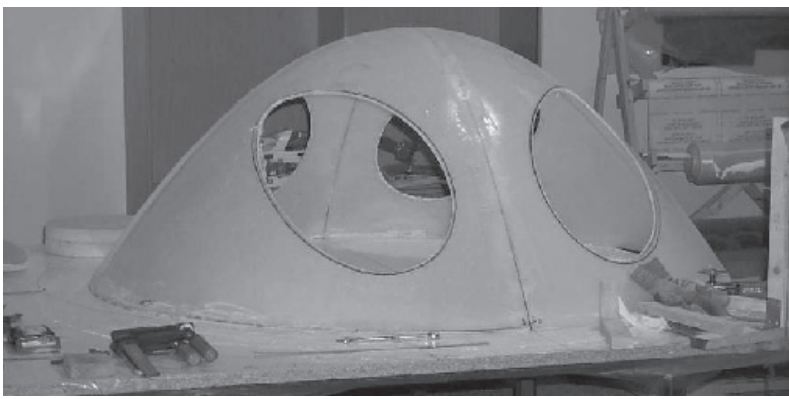

Vorbereitung zum Herausschneiden der Thurstertunnel.

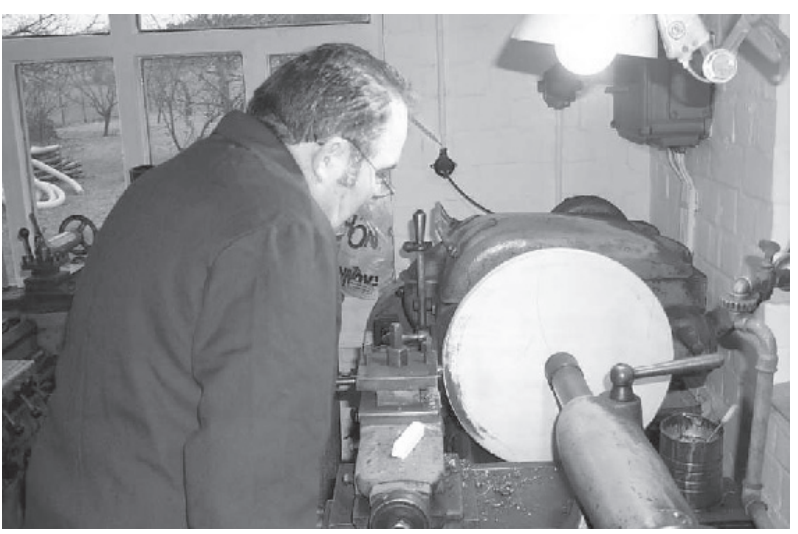

Dies geschieht mittels einer dafür gedrehten Kreisschablone (400 mm). Die Schablone wird auf ein Rohr geschoben, welche die Mittelachse der Thurstertunnel darstellt. Somit wird eine Symmetrie der Ausschnitte gesichert.

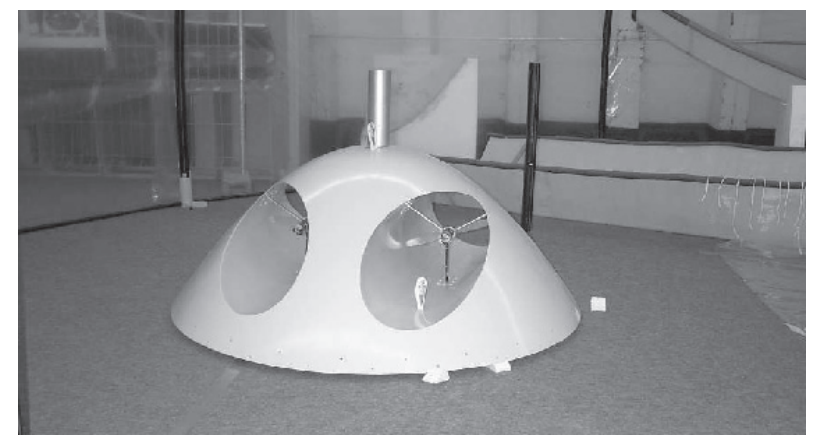

Fertige Nose Cone mit eingebauten Thursterchannels, Motor- mit Motorhalterung und Propellern
Nicht nur die Nose Cone konnte begonnen werden, auch mit den Fins wurde angefangen.

\section{Fins / Leitwerke}

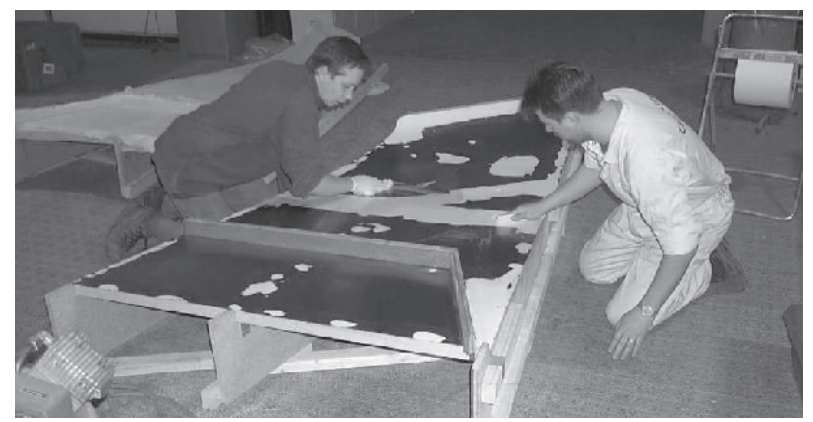

Vorbereiten der Finnenform zum Laminieren (reinigen, wachsen, Trennlack aufbringen).

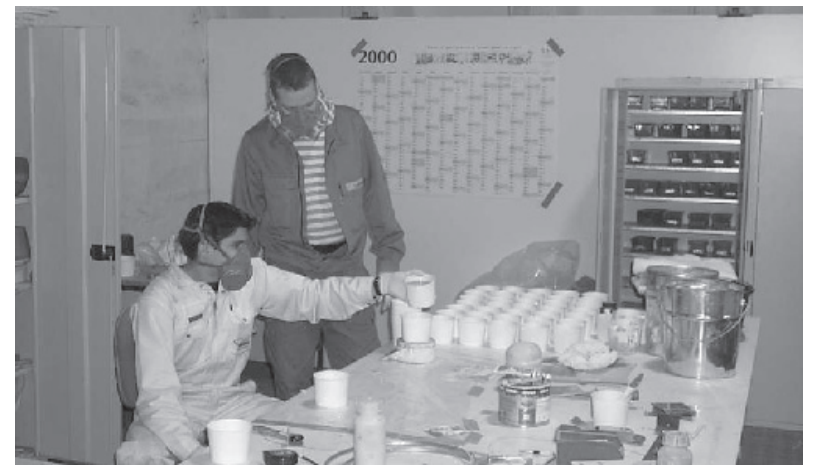

Verhältnisse Harz/Härter abwiegen.

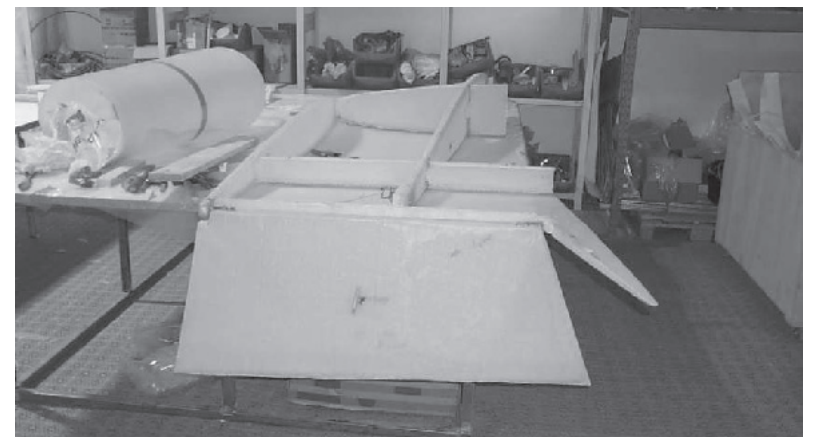

Fertig laminierte Finne mit Holm, Rippe und Ruder.

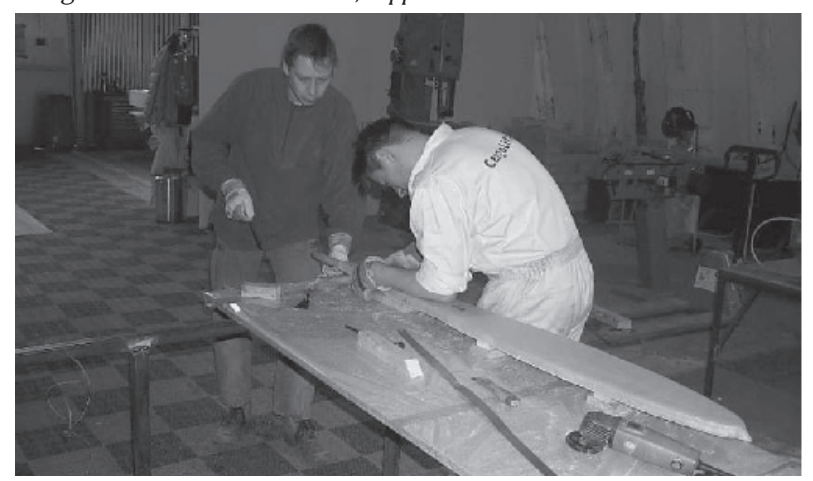

Ausformen einer Rippe.

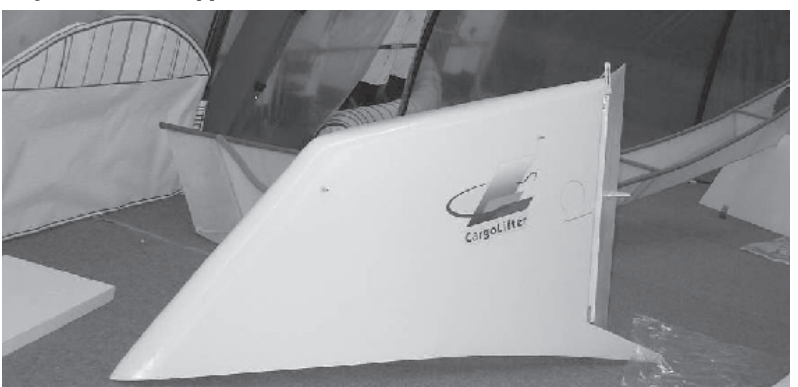

Komplett fertige Finne. 
Doch der positive Eindruck täuschte nicht über die Tatsachen hinweg. Wir stellten die Fakten gegenüber und stellten fest, dass unser Fortschritt subjektiven Charakter besaß. Auch wenn wir weiter mit diesem persönlichen Engagement und dem Engagement der Ingenieure arbeiten würden, wäre unser zeitliches Ziel nicht erreichbar.

\section{Hangarnachbau}

Mit dem Beginn der praktischen Arbeit verdeutlichte sich die Intensität der einzelnen Arbeitspakete immer mehr. Auch die verspätete Fertigstellung der Kranbahn um eineinhalb Wochen spielte ein Rolle. Der Nachbau des Hangars stand unmittelbar mit dem Verzug der Kranbahnendmontage in Zusammenhang. Es konnte nicht vorher mit dem Bau des Hangars begonnen werden.

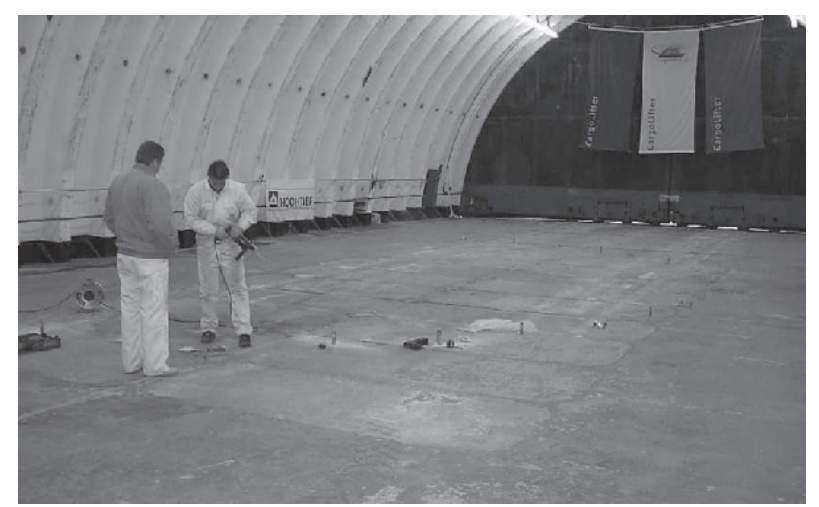

Vorbereitung der PE-Rohre zum Biegen.

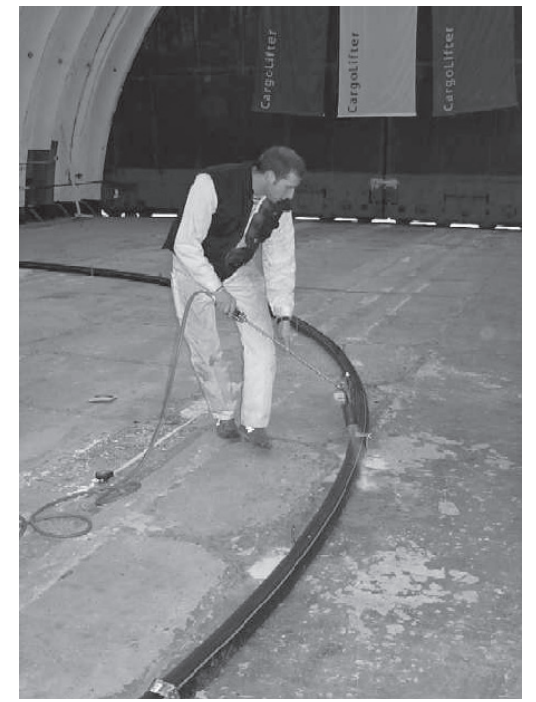

PE-Rohre werden gewärmt und in Bogenform gebracht.

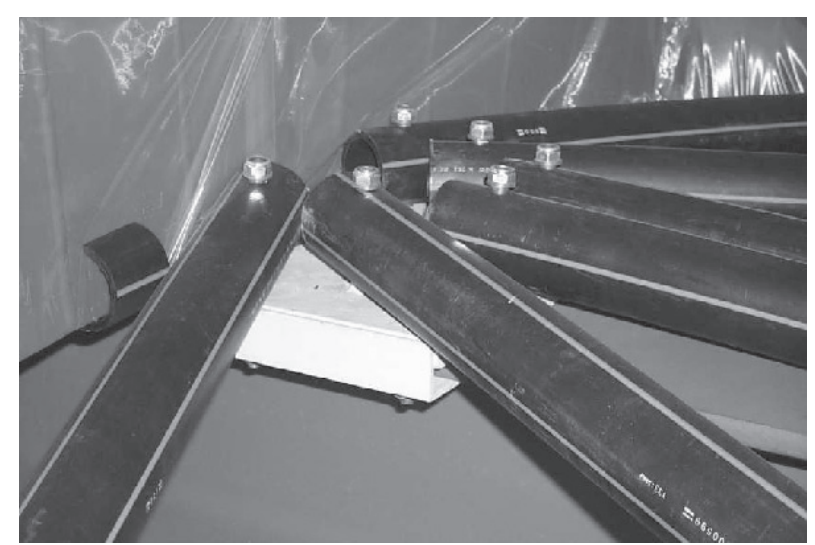

Königszapfen.

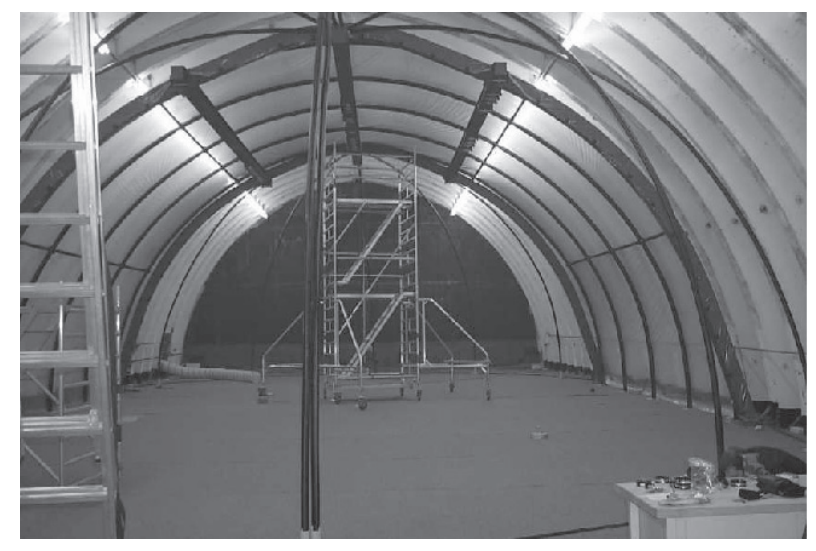

Hangarrohbau, fertig zum Bespannen mit Folie.

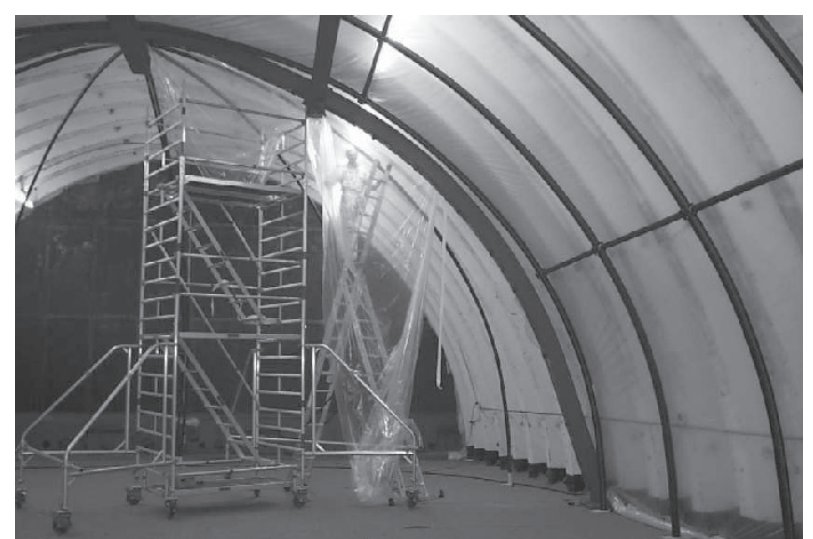

Hangar wird mit Folie bespannt.

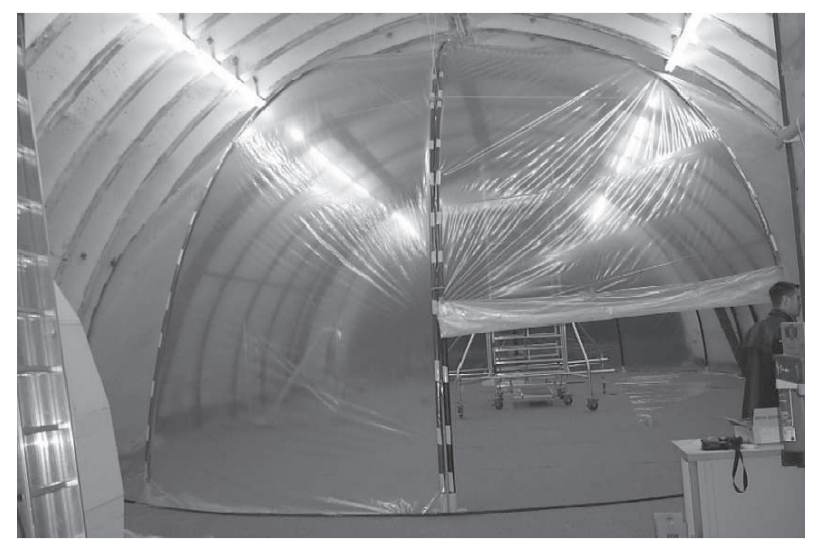

Hangar komplett mit Folie bespannt.

\section{Meeting zum Jahreswechsel}

Im Meeting zum Jahreswechsel 1999/2000 legten wir die gewonnenen Fakten vor. Wir verdeutlichten dies mittels eines Diagramms. Daraus war ersichtlich, dass sich ein deutlicher zeitlicher Verzug abzeichnete und es galt, diesen Verzug zu stoppen.

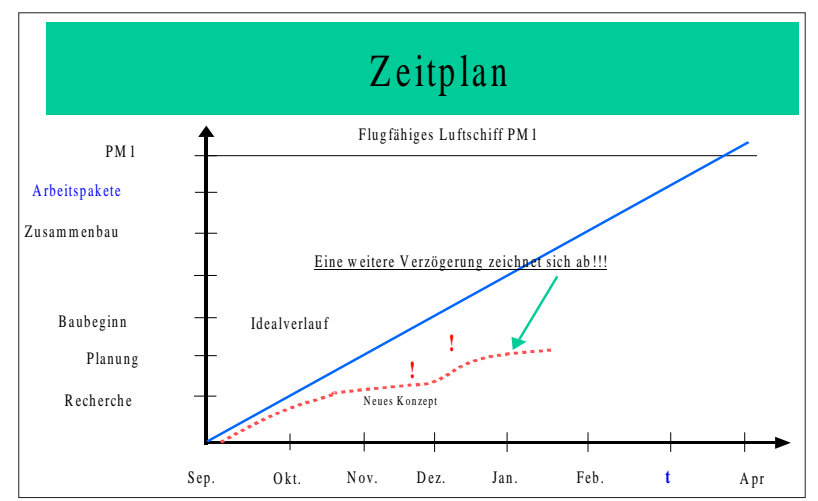


Nach kurzer intensiver Diskussion und gemeinsamer Auswertung unserer Ist-Daten legten wir fest, dass mehr Manpower benötigt wird. Das ist die wesentliche Voraussetzung zum Erreichen unseres zeitlichen Ziels. Nun stellte sich uns die Frage, woher wir die zusätzlichen Arbeitskräfte beziehen sollten? Unter der Berücksichtigung unseres Budgets wägten wir mehrere Möglichkeiten ab:

1. Zeitarbeitskräfte aus Zeitarbeitsfirmen,

2. externe Firma,

3. Praktikanten.

Das Problem, was sich wie ein roter Faden durch das ganze Projekt hindurch zieht, ist der Faktor Zeit. Wir mussten in weniger als 3 Wochen die Arbeitskräfte zur Verfuigung haben. Der Praktikantenbetreuer übernahm die Rekrutierung der Arbeitskräfte persönlich. Punkt 1 und 2 schieden aus unterschiedlichen Gründen aus, so dass die Variante „Praktikanten“ favorisiert wurde.

\section{Trainico-Klasse}

Trainico ist ein Unternehmen, welches die Umschulung/ Qualifikation zum Flugzeuggerätemechaniker-Elektroniker für die Luftfahrt übernommen hat. Die Umschulung dieser speziellen Klasse ist ein Pilotprojekt mit dem Arbeitsamt Cottbus und der Firma CargoLifter. Sie werden fuir den Einsatz bei der Montage vom CL-Luftschiffen vorbereitet. Zum Erstaunen unseres gesamten Teams hatten alle 15 Klassenmitglieder ihr Interesse angemeldet, am PM1 mitzuwirken.

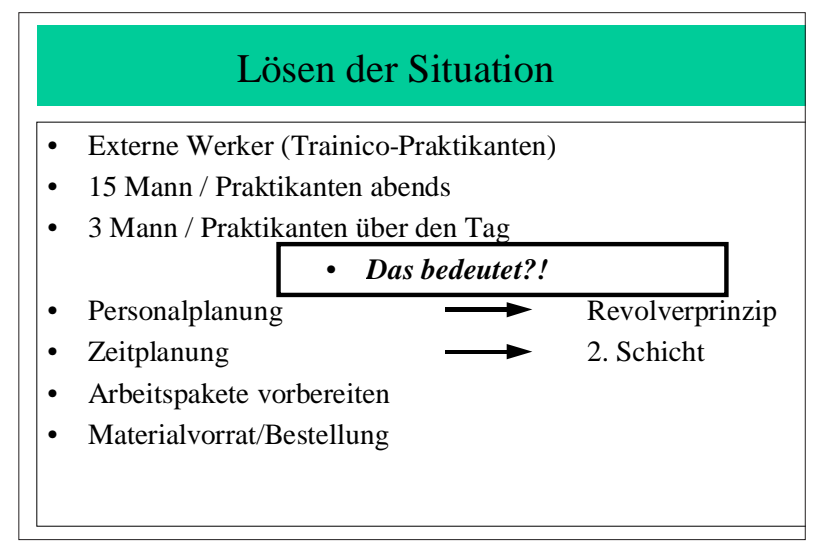

Als weitere Herausforderung stellte sich für uns die Aufgabe, die neuen Mitarbeiter (Trainico-Leute) in das laufende Projekt reibungslos zu integrieren. Dies bedeutete die Planung der Einarbeitung und das vorbereiten der Arbeitspakete für die neuen Kollegen.

\section{Personalplanung}

An erster Stelle stand die Personalplanung, da wir nicht 15 Werker von heute auf morgen einarbeiten konnten. Wir entschieden uns für den rollierenden (Revolverprinzip) Personaleinsatz. Hinter dem rollierenden Personaleinsatz verbirgt sich nichts anderes, als dass wir mit der Einarbeitung von 3 Trainico-Leuten anfingen und diese in der darauffolgenden Woche ihre Kollegen selber einarbeitetn. So entstand ein Turnus von jeweils 2 Wochen Anwesenheit pro Trainico-Mann/Frau. Der Vorteil dabei war, dass sich ein Synergieeffekt (Eigendynamik) in der Klasse entwickelte und dass wir darüber hinaus von der erneuten Einarbeitung neuer Leute entbunden waren. Wir konnten uns verstärkt auf die Überwachung, Kontrolle und Organisation des gesamten Projektes konzentrieren. Die Ergebnisse zeichneten sich unmittelbar im Projektplan nieder.

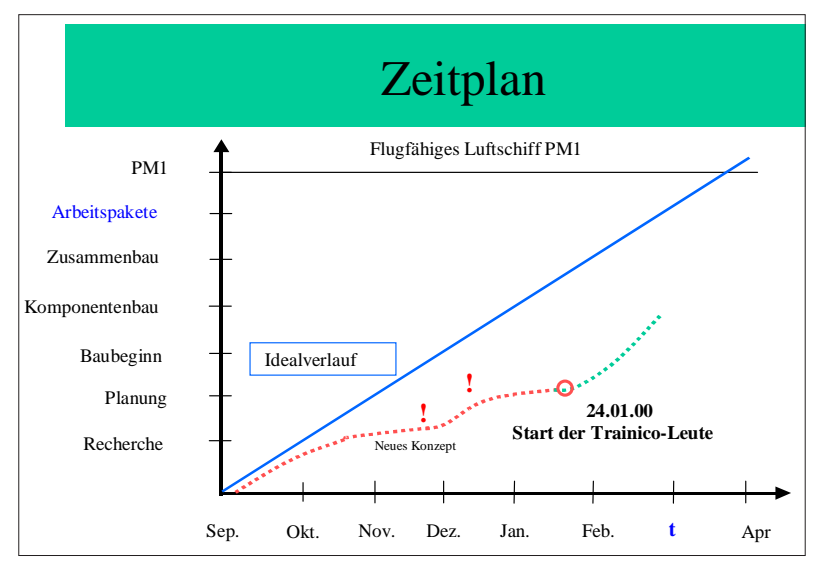

\section{Keel / Kiel}

\section{Welche Arbeitspakete kamen auf uns zu?}

Eine der arbeitsintensivsten Komponenten des gesamten Luftschiffes war der Nachbau des Kiels. Der Kiel ist in sieben Segmente unterteilt worden. Er erstreckt sich über eine Länge von fast $15 \mathrm{~m}$ und überwindet dabei eine Höhendifferenz von ca. $2 \mathrm{~m}$. Es ist das Teil mit dem höchsten Materialverbrauch des gesamten Modells. Die Arbeit gliederte sich vom Formbau bis hin zum Spachteln und Lackieren der Sichtflächen.

In einem kurzen Zusammentreffen mit den System-Ingenieuren klärten wir ab, aus welchen Materialien die Kielformen gebaut werden sollten. Dabei wurde auch direkt festgelegt, ob es eine Positiv- oder Negativ-Form werden würde. Fünf der Segmente sind Negativ-Formen, die beiden übrigen Positiv-Formen. Als das entschieden war, konnte anhand von Skizzen ein Materialbedarf und die daraus folgenden Angebotsschreiben erstellt werden. Dies geschah in enger Zusammenarbeit mit der Abteilung Einkauf. Die Negativ-Formen wurden aus Holzlatten, Spanplatten, Blech und einer Unmenge von Holzschrauben zusammengefuigt. Die beiden Positiv-Formen sind aus Styroporblöcken geschliffen worden. Um die genauen Formen herzustellen, ließen wir uns die Profile durch das CAD-Labor ausdrucken. Diese brauchten dann nur noch ausgeschnitten zu werden und dienen als Vorlage. Die Profilausschnitte legten wir auf die Spanplatten bzw. die Styroporblöcke und zeichneten die Konturen ab. So entstanden die Linien, die wir für den Ausschnitt mit Handstich- und Bandsäge benötigten.

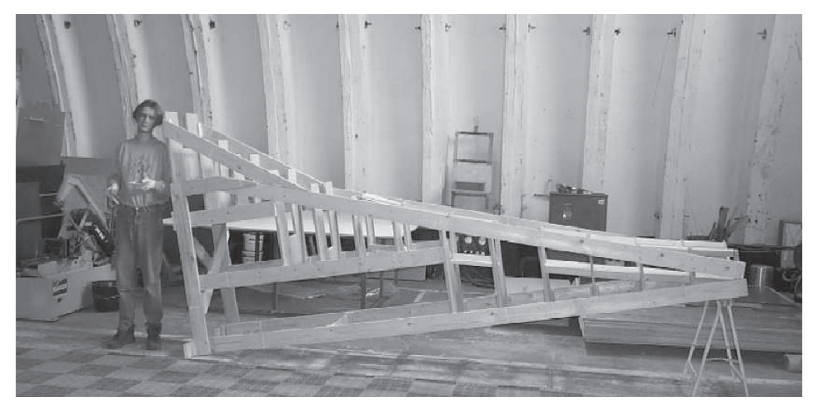

Grundstruktur eines Kielformsegmentes 


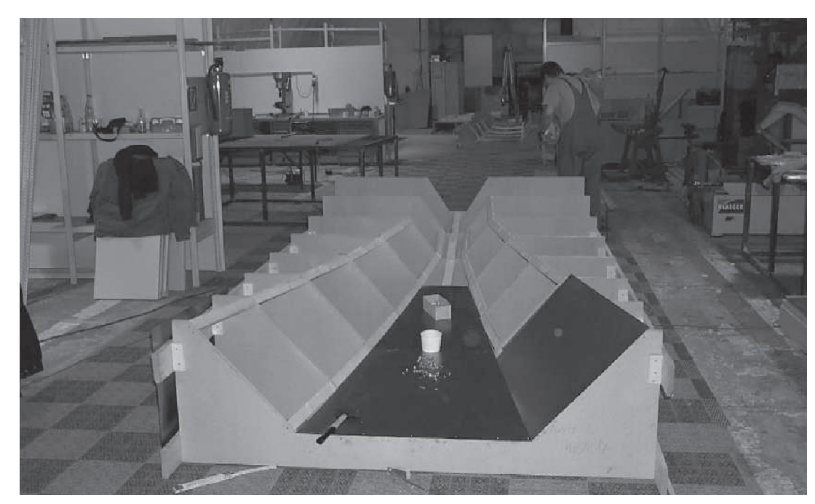

Einlegen der Bleche.

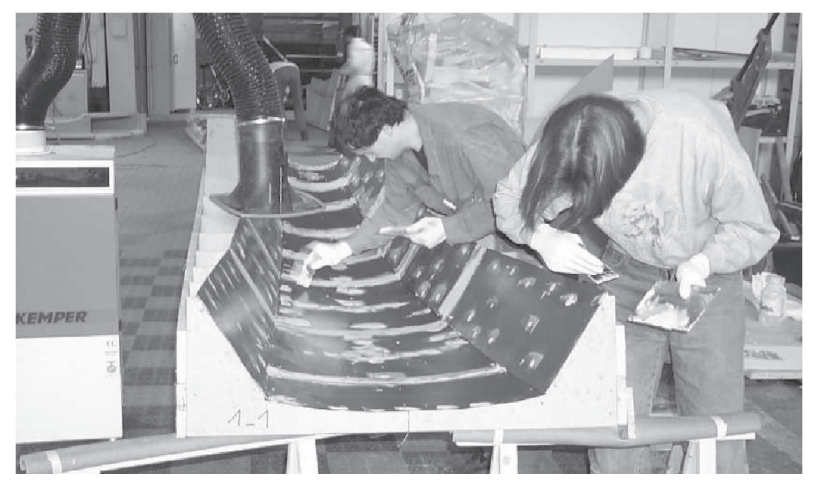

Spachteln der Form vor dem Laminieren.

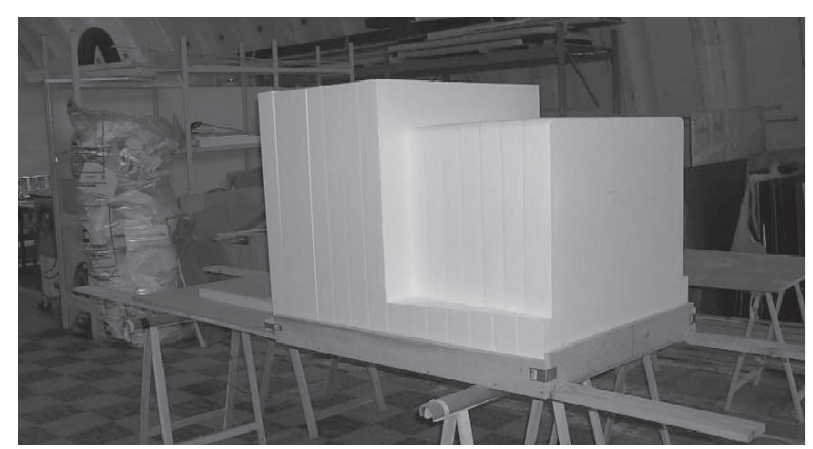

Grobausschnitt der Positivform aus Styropor.

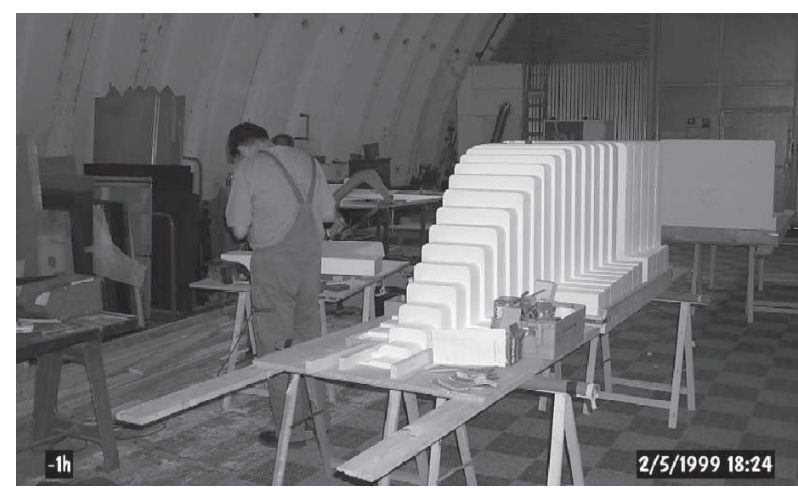

Profilausschnitt / Konturen sind erkennbar.

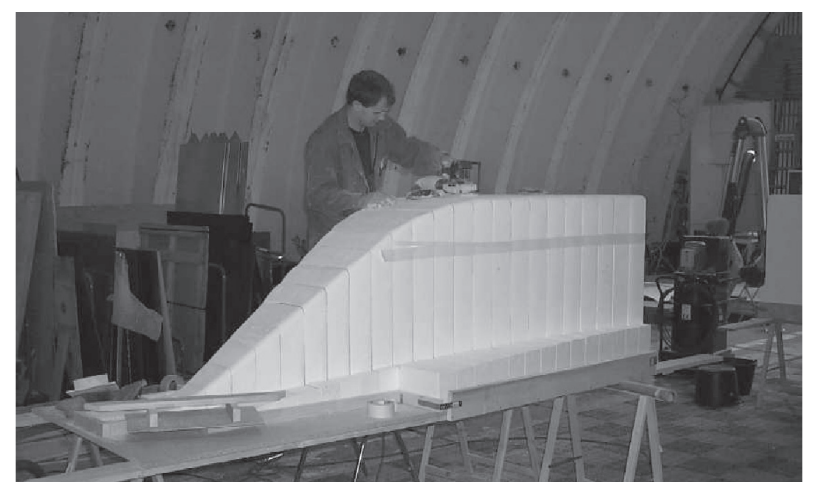

Feinschliff der Form.

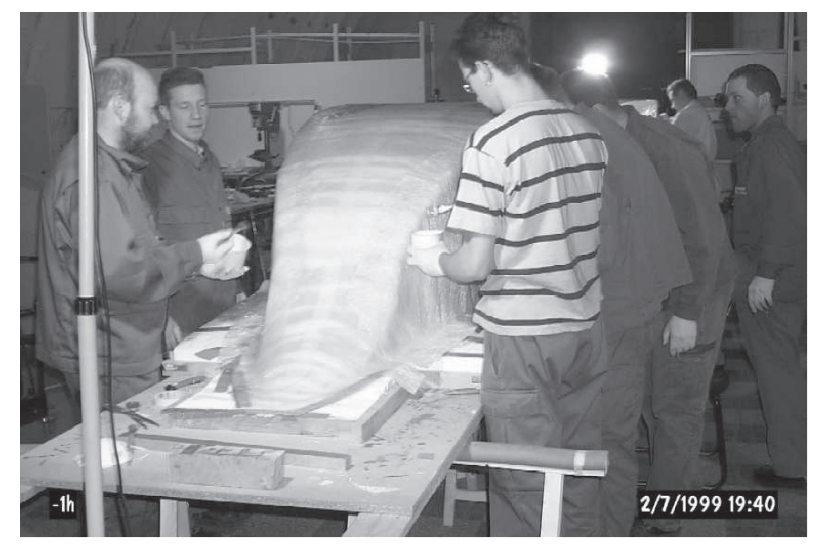

Laminieren der Form.

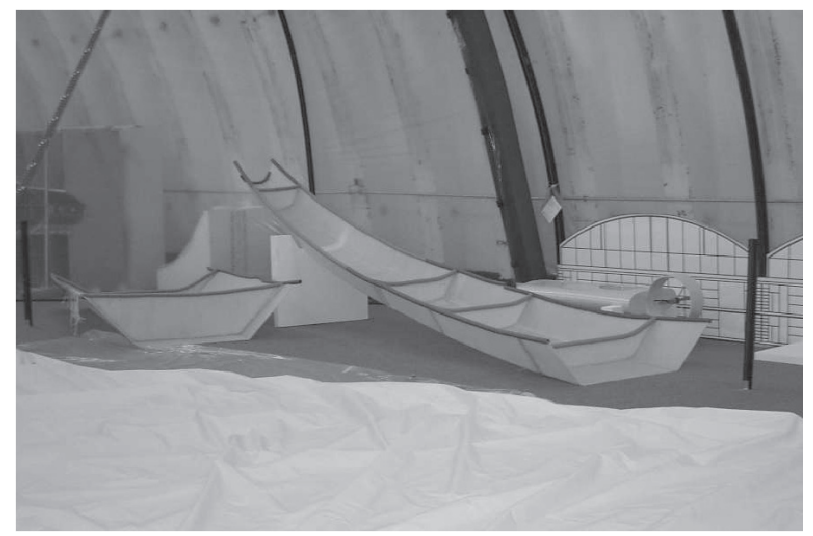

Vorderes Teil eines fertigen Segmentes.

\section{Powerwings}

Die Powerwings beherbergen zwölf Turbinen. Zum einen die Antriebe, um sich in der Vertikalen zu bewegen, und zum anderem die an den Enden der Wings montierten Marschtriebwerke. Die Propeller haben im Original einen Durchmesser von $6 \mathrm{~m}$.

Beim Nachbau dieser Komponente legte der System-Ingenieur selber mit Hand an. Angefangen vom Ausschneiden der Rippen, Vernieten Rippe/Holm, Laminieren der Außenhaut bis hin zum Bauen der einheitlichen Motorhalterungen. Wir konnten sehr auf seine praktische Erfahrung aus dem Luftfahrtbereich zurückgreifen und diese mit in das Projekt einfließen lassen.

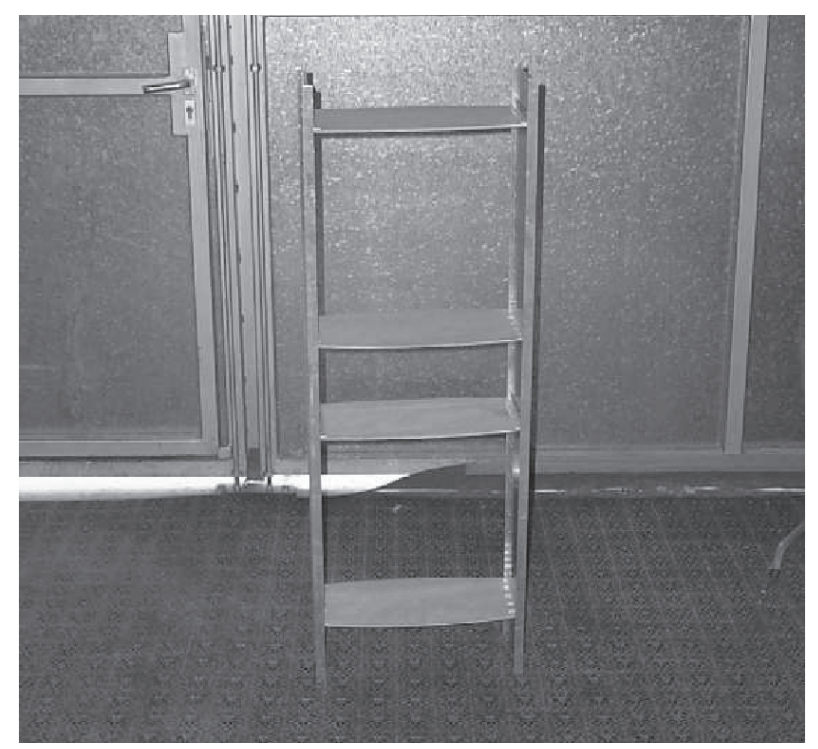

Trägerstruktur / Powerwing 


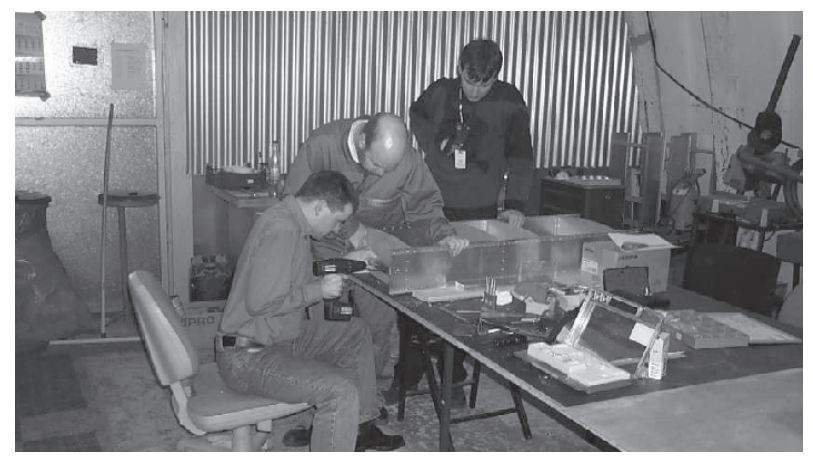

Vorbereitung zur Montage des Ducts / Marschantrieb
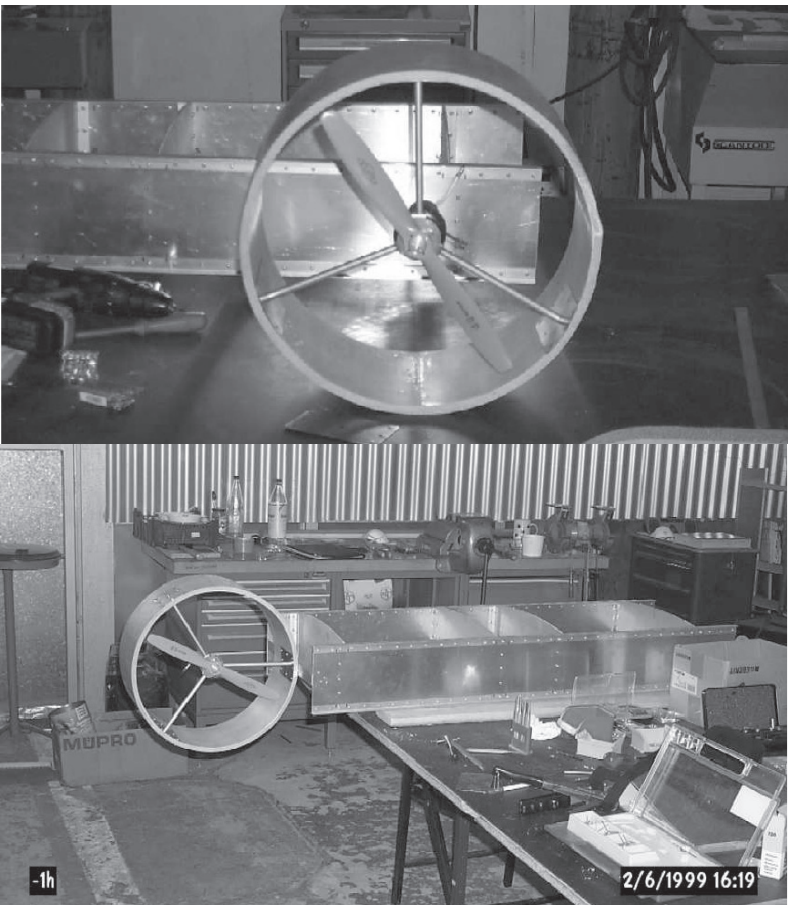

Antrieb / Duct montiert

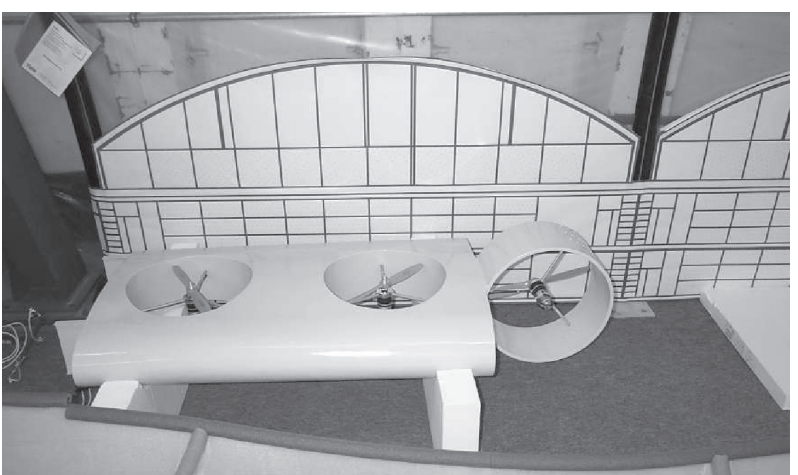

Fertiger Powerwing mit Bespannung und lackiert

\section{Hülle / Envelope}

Die Hülle ist die einzige Komponente, die wir nicht selber gefertigt haben. Das Design der Hülle ist in enger Zusammenarbeit mit unserem CAD-Labor und in Abstimmung mit den System-Ingenieuren entwickelt worden. Wir bezogen uns auf die altbekannte Form von Luftschiffen, die mittels der Gertler-Formel errechnet wird. Nach Festlegung aller fuir uns wichtigen Features sendeten wir es zur Fertigung nach England. Wir bedienten uns dabei des Datentransfers via Internet. Die Hülle ist aus einem heliumdichten Material gefertigt. Sie ist 16,25 m lang, besitzt ein Volumen von ca. 150 $\mathrm{m}^{3}$ und wird mit einem Innendruck von 450-650 $\mathrm{Pa}$ versehen sein.

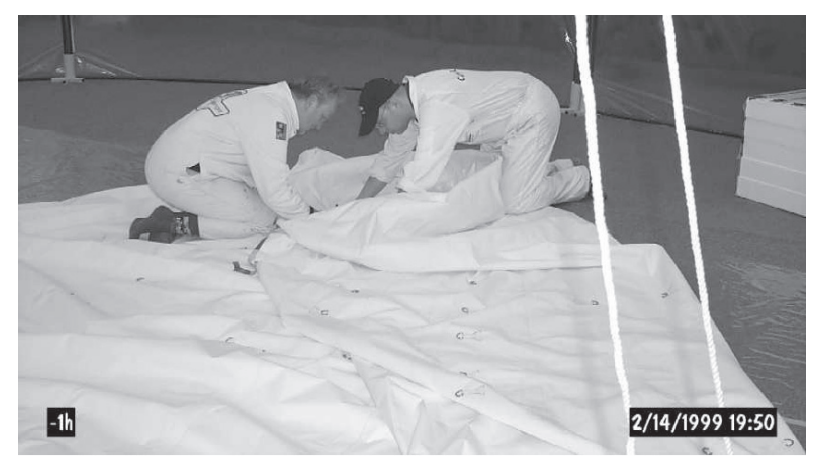

Letzte Arbeiten an der Hülle

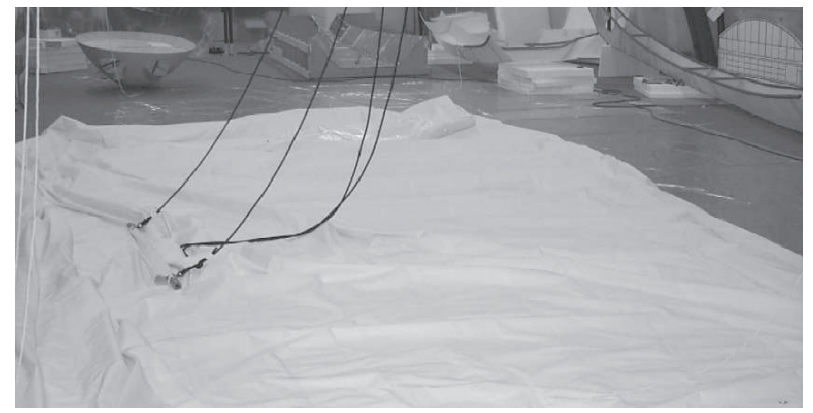

Hülle zum Befüllen mit Luft vorbereitet

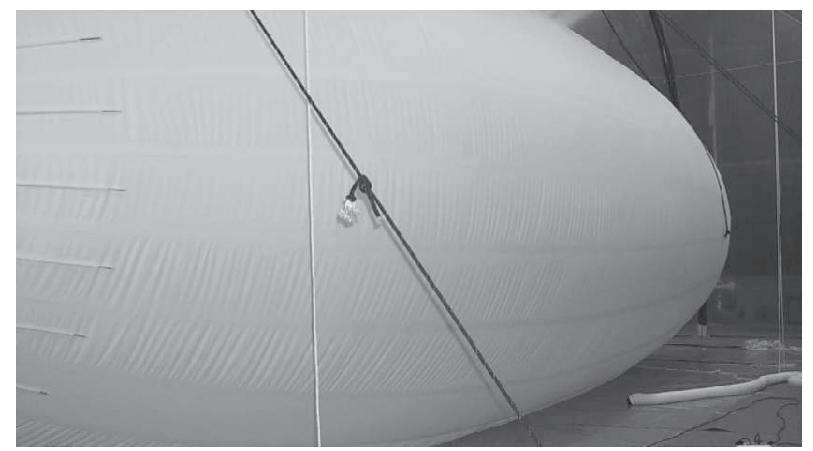

Hülle prall aufgepumpt (650 Pa)

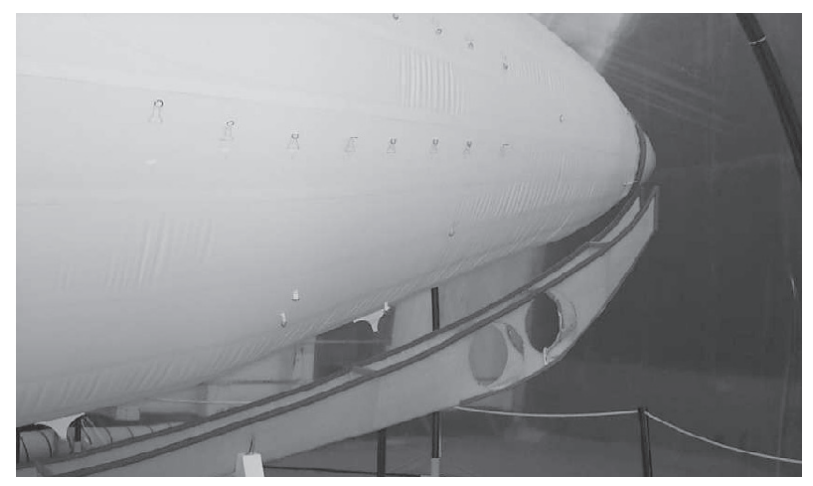

Hülle und Kiel zum „Verheiraten“ vorbereitet

\section{Laderahmen / Loadframe}

Die Maße des Modell-Laderahmens besitzt eine Länge von $3 \mathrm{~m}$ und eine Breite von 0,5 $\mathrm{m}$. Im Original ist er 50 $\mathrm{m}$ lang und $8 \mathrm{~m}$ breit und wird 36 Container transportieren können. Der Nachbau unseres 1:16 Laderahmens erfolgte aus Aluminium. Die Platte entstand aus Abstandgewebe. Die Zuschnitte wurden verschweißt. In dem gesamten Projekt kamen die unterschiedlichsten Herstellungsverfahren zum Einsatz. Angefangen vom Laminieren, Kleben, Spachteln, Tempern, Lackieren, Schweißen bis hin zum Nieten. Nicht zu vergessen die funktionsfähigen Wassertanks, die für das Lastaustauschverfahren unabdingbar sind. 


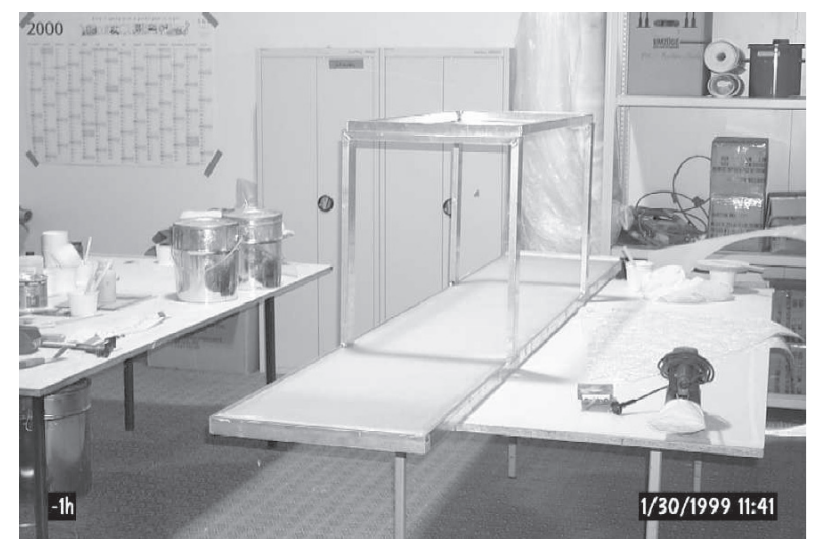

Laderahmen verschweißt und mit Ladeplattform versehen.

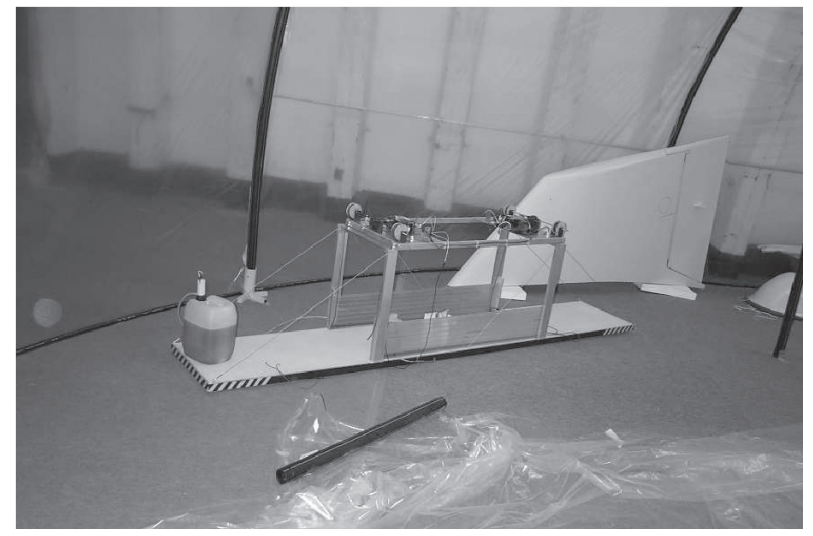

Rahmen komplett fertig mit Windenrahmen und Wassertanks.

\section{Hauptversammlung}

Der Tag der Aktionärshauptversammlung rückte immer näher. Dieser Tag war die Deadline für unser Projekt. Im November 1999 wurde festgelegt, dass PM1 zur Hauptversammlung zu präsentieren ist. Das stellte für uns die Herausforderung dar. Wir konnten den Aktionären mit unserem Projekt die Dimensionen offenlegen. Bis dato waren es nur Computeranimationen, nichts Richtiges zum anfassen.

Das Luftschiff stellte eine Attraktion der Hauptversammlung dar. Wir organisierten das gesamte Projekt so, dass die Aktionäre durch den Hangar gehen konnten. An jeder Komponente des Luftschiffes stand ein Mitarbeiter, um die vielen Fragen der Aktionäre beantworten zu können. Die positive Resonanz der Besucher war eine weitere Belohnung für die Anstrengungen der letzten Tage und Wochen und bestätigte das Unternehmen in seinem Konzept.

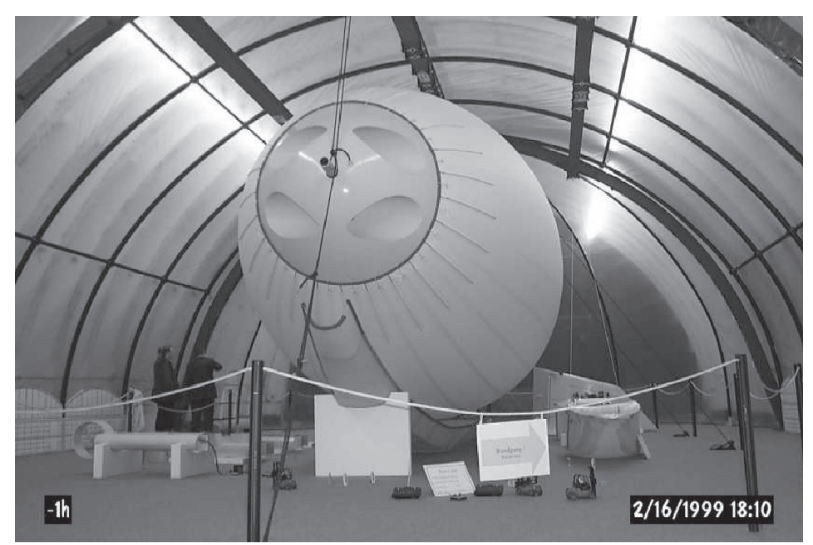

Frontansicht des Luftschiffes

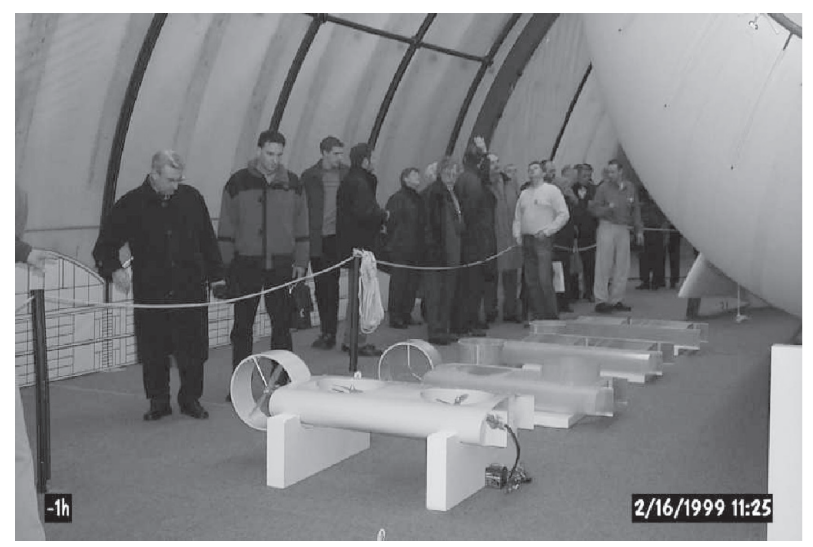

Bauabschnitte der Powerwings werden den Besuchern erklärt.

\section{Robby}

Robby ist ein Jugendlicher im Alter von 16 Jahren aus dem Kinderdorf Rankenheim bei Groß Köris. In dem Jugenddorf in Groß Köris leben überwiegend verhaltensauffällige Kinder und Jugendliche. 56 Bewohner im Alter zwischen 12 und 17 Jahren hat das Heim. Robby gehört mit seinen 16 Jahren schon zu den „Alten“. Er begleitete uns im Rahmen eines freiwilligen Praktikums vom November 1999 bis jetzt 2 mal pro Woche nach seinem Schulunterricht. Dies ist ein Programm, welches durch den Einkaufsleiter, den Director of Production Herrn Christoph von Kessel und die Sozialbetreuerin (Kinderdorf Rankenheim) Frau Dörring ins Leben gerufen wurde.

Wir waren mit der Betreuung beauftragt worden. Der Zweck dieses Projektes ist es, Robby eine Möglichkeit zu geben, wieder in die Gesellschaft mit einbezogen zu werden, seine Fähigkeiten und Teamgeist ausfindig zu machen und zu fördern. Dies geschah durch praktische Mitarbeit am Projekt sowie die mündliche und schriftliche Begleitung seiner Tätigkeit. Darunter kann man sich sowohl seine Tagesberichte, als auch die mündliche Auswertung am Ende bzw. während seiner Arbeitszeit vorstellen. Der Projektverlauf ist von beiden Seiten als sehr positiv eingeschätzt worden. Durch den ständigen Kontakt mit dem Kinderdorf sind keine Schwierigkeiten aufgetreten. Für Robby eröffnete sich die Möglichkeit, im Rahmen seiner Schulausbildung sein Schülerpraktikum dort absolvieren.

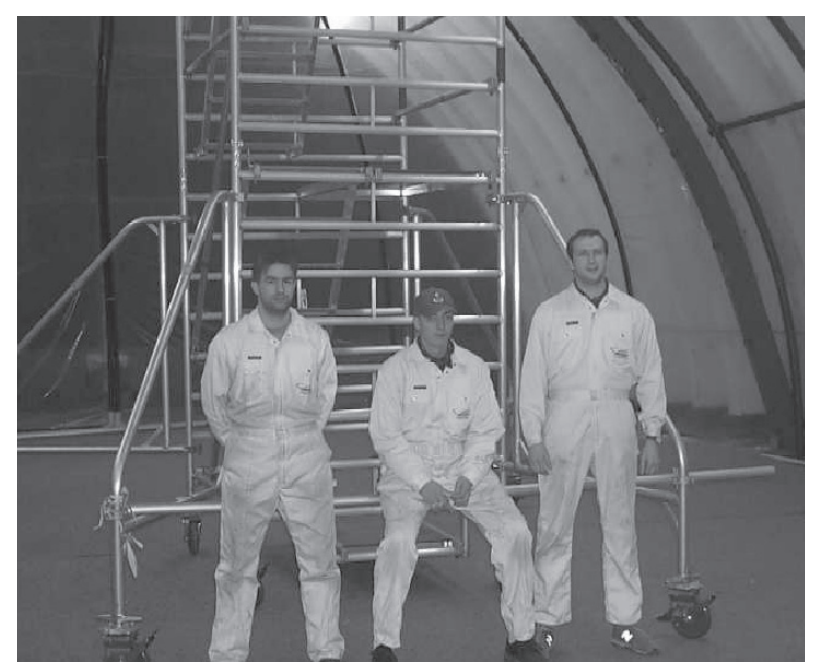

v.l.n.r.: Marco Heyn, Robby Meyn, Stephan Wojcik 


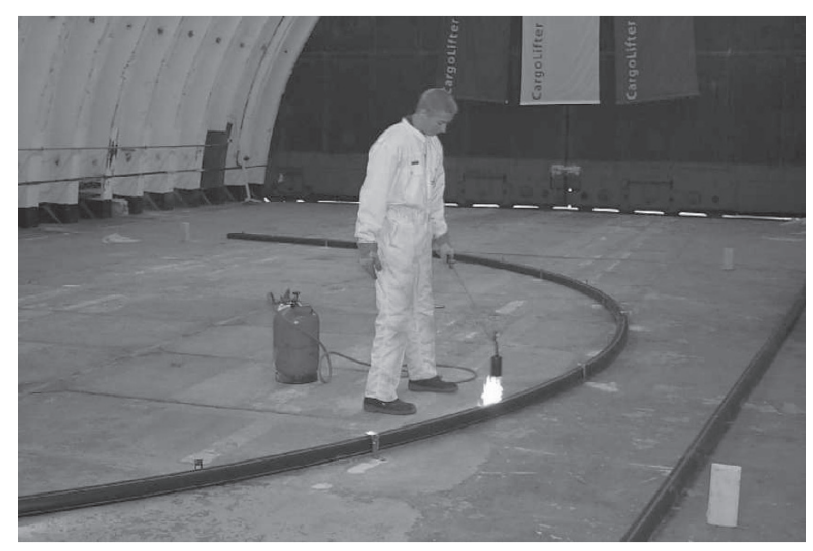

Robby beim Wärmen des PE-Rohrs.

\section{Resümee}

Wenn die Gedanken zum Anfang unseres Praktikums zurïckgehen, so wird das Geleistete erst richtig bewusst. Die uns übertragende große Verantwortung und das daraus resultierende freie Handeln gab uns die Möglichkeit, unserer Kreativität und unserem Engagement freien Lauf zu lassen. Wir konnten das uns vermittelte theoretische Wissen aus den Vorlesungen in die Praxis umsetzen und wurden in die Lage versetzt, unsere Dozenten noch besser zu beurteilen.

Den Beginn des Praktikums möchten wir mit einer „Black Box“ vergleichen, welche erst mit Inhalt gefuillt werden musste.

Dies bedeutet, dass diese klaren Strukturen, wie sie am Ende bestanden haben, uns nicht von Anfang an zur Verfuigung standen. Diese wurden im Laufe des Voranschreitens des Projekts von uns mit Unterstuitzung der System-Ingenieure erarbeitet. Wir waren in fast allen Bereichen des Unternehmens integriert, angefangen vom Einkauf, Controlling, Produktion, Design bis hin zur Personalabteilung.

Die gute Arbeitsatmosphäre trug einen wesentlichen Teil zum Gelingen des Projektes bei.

Es kann gesagt werden, dass wir in unserem Praktikum mit dem realen Projektmanagement konfrontiert waren. Beginnend mit der Aufstellung des Idealzeitplanes bis hin zum ständigen Abgleichen des Planes und Finden von neuen Lösungswegen.

Das positive Feedback und die Anerkennung der Kollegen aus dem gesamten Unternehmen unterstreicht das Resultat unserer geleisteten Arbeit.

\section{Autoren}

Prof. Dipl.-Ing. Alfred Hentschel

Technische Fachhochschule Wildau

Fachbereich Ingenieur-/Wirtschaftsingenieurwesen

Labor Produktionstechnik/Produktionsvorbereitung

Tel. (0 33 75) 508-117, -133

E-Mail: hentschel@igw.tfh-wildau.de

\section{Marco Heyn}

Studiengang Wirtschaftsingenieurwesen/

Produktionsmanagement

Tel. (03 37 66) 41545

E-Mail: maheyn@gmx.net

\section{Stephan Wojcik}

Studiengang Wirtschaftsingenieurwesen/

Produktionsmanagement

Tel. (03 37 63) 62302

E-Mail: wojcik@gmx.net 